\title{
Kinetics of one-dimensional swelling and shrinking of polymer gels under mechanical constraint
}

\author{
Atsushi Suzukia) and Taku Hara \\ Department of Materials Science, Yokohama National University, 79-5 Tokiwadai, \\ Hodogaya-ku, Yokohama, 240-8501, Japan
}

(Received 11 September 2000; accepted 2 January 2001)

\begin{abstract}
The swelling and shrinking kinetics of thin-plate poly $(N$-isopropylacrylamide $)$ gels with rectangular surfaces under mechanical constraint was investigated. The top and bottom surfaces were chemically clamped on the glass plates, and the gel could swell and shrink only along the thickness direction between two swollen states by the temperature jumps. The time evolution of the thickness was well described by a single exponential except immediately after the temperature change. The characteristic time was found to depend on the lengths of the constrained surfaces and not on the thickness at the time of sample preparation. The swelling and shrinking kinetics of the one-dimensional thickness direction is essentially governed by the relaxation of the polymer network in the two-dimensional direction along the surface. (c) 2001 American Institute of Physics.
\end{abstract} [DOI: $10.1063 / 1.1350576]$

\section{INTRODUCTION}

It has been well established that the kinetics of gel swelling and shrinking is governed by the collective diffusion of the polymer network relative to the solvent. ${ }^{1}$ The kinetics is related to the elasticity (bulk modulus $K$ and shear modulus $\mu)$ as well as the friction $f$ between the polymer and the solvent. ${ }^{1-7}$ In accordance with the kinetic theory of the collective diffusion of gels, a single exponential decay with time has been experimentally confirmed, which is valid for spherical, cylindrical, and disk gels. ${ }^{1-3}$ The macroscopic kinetics can be described by

$$
\frac{\partial \mathbf{u}(\mathbf{r}, t)}{\partial t}=D \nabla^{2} \mathbf{u}(\mathbf{r}, t),
$$

where $\mathbf{u}$ is the displacement of a point $\mathbf{r}$ on the polymer network at time $t$ from its average location and $D=(K$ $+4 \mu / 3) / f$ is the collective diffusion constant of the polymer network. This equation predicts that the relaxation time $\tau$ of a spherical gel is proportional to the square of the diameter $d$ and is inversely proportional to $D: \tau \propto d^{2} / D$. For cylindrical and disk-like gels with symmetrical geometry, the theory was extended by taking the shear relaxation into consideration. ${ }^{7}$ These expressions have been used to analyze kinetic properties when the motion of the polymer network solely governs the kinetics in response to a change in an environmental condition. The square-power law has been experimentally investigated on the thermoresponsive $\operatorname{poly}(\mathrm{N}$ isopropylacrylamide) (NIPA) gels. ${ }^{2,6}$ This investigation is reasonable since the thermal diffusion is much faster than the collective diffusion of the polymer network. Although a power smaller than two and the initial deviation from the single exponential function have been reported, ${ }^{2,6}$ almost all previous investigations have been discussed on the basis of this collective diffusion relative to the solvent. The experi-

\footnotetext{
a) Author to whom all correspondence should be addressed; electronic mail: suzuki@post.me.ynu.ac.jp
}

mental results are qualitatively consistent with the theory, which is related to the fact that the swelling and shrinking of gels are isotropic and the gel can freely swell and shrink along the three-dimensional direction. So far no experimental observation has been reported on the anisotropic swelling and shrinking of gels.

In this paper, we report for the first time the kinetics of thin-plate NIPA gels with rectangular surfaces under mechanical constraint. The swelling and shrinking were restricted along the two-dimensional direction along the surface; the gel could only swell and shrink along the onedimensional thickness direction. The time evolution of the thickness between two different swollen states at 11.5 and $30{ }^{\circ} \mathrm{C}$ was measured by abruptly changing the temperature. We investigated the effects of the gel sizes (two surface lengths and thickness) on the swelling and shrinking kinetics.

\section{EXPERIMENTAL METHOD}

The gels were prepared at the ice temperature by a free radical polymerization reaction ${ }^{8}$ in water between two glass plates (slides) separated by thin spacers. The pregel solution was the mixture of purified NIPA (main constituent, $7.8 \mathrm{~g}$, Kohjin), $N, N^{\prime}$-methylenebisacrylamide (cross-linker, 0.133 g, Wako), and $N, N, N^{\prime}, N^{\prime}$-tetramethylethylenediamine (accelerator, $240 \mu \mathrm{l}$, Wako), which were dissolved in pure water (100 g, deionized and distilled water). After being fully saturated with nitrogen, the pregel solution was stored at the ice temperature, and ammonium persulfate ( $0.04 \mathrm{~g}$, Wako) was added to the solution to initiate the polymerization. The pair of two glass plates (slides for an optical microscope 26.0 $\times 76.0 \mathrm{~mm}^{2}$ ) was immediately inserted into the solution. The pregel solution was brought into the thin space by capillary action. In order to chemically clamp the gel on the slides Bind Silane (Pharmacia) was used to rinse both slides before they were dipped into the pregel solution. ${ }^{8}$ The slides were silanized by the Bind Silane, $\mathrm{CH}_{2}$ $=\mathrm{C}(\mathrm{O}) \mathrm{C}\left(\mathrm{CH}_{3}\right) \mathrm{OCH}_{2} \mathrm{CH}_{2} \mathrm{CH}_{2} \mathrm{Si}\left(\mathrm{OCH}_{3}\right)_{3} \quad(\gamma$-methacryloxy- 


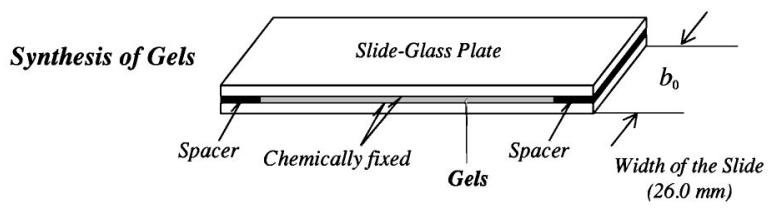

Effects of Surface Length
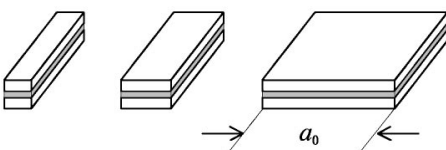

Length $(4.2 \sim 58.9 \mathrm{~mm})$

Effects of Thickness
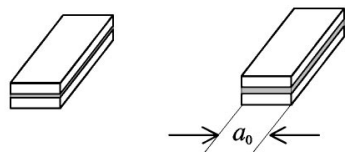

Length (Constant, $9.5 \pm 0.5 \mathrm{~mm}$ )

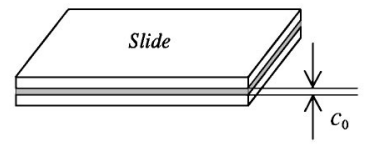

Thickness at gelation (Constant, $0.90 \pm 0.03 \mathrm{~mm}$ )

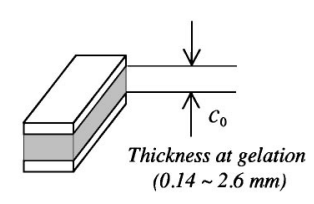

FIG. 1. Schematic illustrations of the thin plate NIPA gels synthesized between two slides. Both surfaces are chemically clamped on the glasses. The samples with different thickness and width were prepared.

propyltrimethoxy-silane), which added the reactive methacrylate groups onto the slide surfaces so that NIPA, $\mathrm{CH}_{2}=\mathrm{CHCONHCH}\left(\mathrm{CH}_{3}\right)_{2}$, became chemically bound to the groups during polymerization. The gelling solution was stored at the ice temperature for at least $12 \mathrm{~h}$ to allow the gelation process to be completed. The clamped gels were subsequently immersed in a large amount of pure water to wash away residual chemicals and unreacted monomers from the polymer network. A sample shape is schematically shown in Fig. 1. To obtain the effects of the surface length, we set one of the surface lengths $b_{0}$ at the width of the slide $(26.0 \mathrm{~mm})$ except in the additional experiments (mentioned later), while changing the other length $a_{0}$ to between 4.2 and $58.9 \mathrm{~mm}$ by cutting the slides together with the gel using a diamond cutter. In this case, the thickness of the gel at gelation $c_{0}$ was almost constant, $0.90 \pm 0.03 \mathrm{~mm}$. To measure the effects of $c_{0}$, on the other hand, several spacers with thickness ranging between 0.14 and $2.6 \mathrm{~mm}$ were used in order to obtain a different $c_{0}$. In this case, $a_{0}$ was almost constant, $9.5 \pm 0.5 \mathrm{~mm}$. The sample was set in a small thermostat bath with water, and the temperature was regulated to within $\pm 0.05^{\circ} \mathrm{C}$. A step-like change in water flow was made between 11.5 and $30^{\circ} \mathrm{C}$ where the circulating initial water temperature was abruptly increased to the final temperatures by switching the water flow from one water bath to another. ${ }^{9}$ The thickness of the gel $c$ was measured by an optical microscope, connected to a calibrated charge coupled device apparatus.

\section{RESULTS AND DISCUSSION}

When the external temperature was rapidly changed, $c$ gradually approached to the equilibrium value. The time evolutions of the normalized thickness $c / c_{0}$ on swelling processes are shown in Fig. 2(a) for the gels with different $a_{0}$ values, and in Fig. 2(b) for the gels with different $c_{0}$ values. One can see that the swelling velocity becomes smaller with
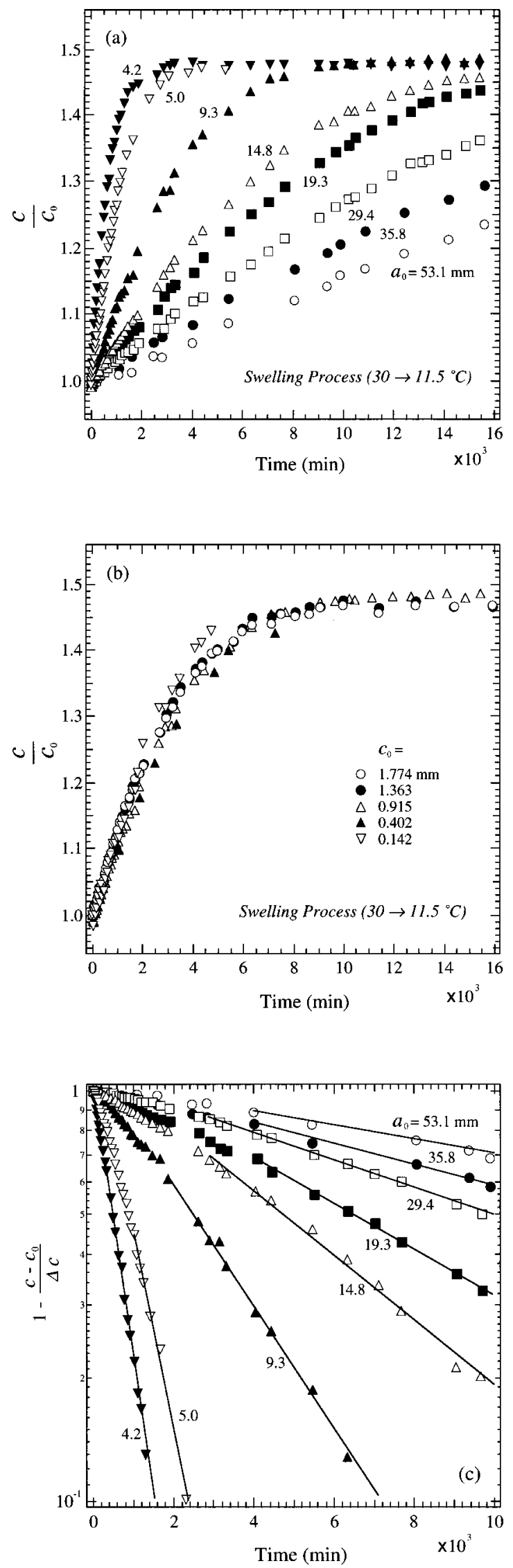

FIG. 2. Time evolution of the normalized thickness $c / c_{0}$ in response to a temperature jump from 30 to $11.5^{\circ} \mathrm{C}$ : (a) for the gels with different $a_{0}$, (b) for the gels with different $c_{0}$, and (c) the normalization of (a) using Eq. (2). The thickness changes exponentially except at the beginning just after the temperature change. 
increasing $a_{0}$, while it does not depend on $c_{0}$. As is shown in Fig. 2(c), the time evolution was found to be welldescribed by a single exponential:

$$
c=c_{I}+\Delta c\left(1-e^{-(t / \tau)}\right),
$$

where $c_{I}$ is the initial thickness (at 11.5 or $30{ }^{\circ} \mathrm{C}$ ), $\Delta c$ indicates the total thickness change $\left(=c_{I}-c_{F} ; c_{F}\right.$ is the final thickness), and $\tau$ represents the characteristic time of swelling or shrinking. Note that the slight deviation from the exponential immediately after the temperature change can be seen and is similar to the results on the gels without mechanical constraint. ${ }^{1,2}$ This could be due to extrinsic factors, such as the excess internal stress introduced by initial sample handling while the glass was being cut, or to intrinsic factors, such as an imperfection in the network structure. ${ }^{10}$ The network structure at the mesoscopic level is constructed of highly cross-linked microgels connected by a loose network. ${ }^{11}$ This unique structure can be introduced during the gelation process, which would affect deviation at the initial stage.

Figure 3(a) shows the effects of $a_{0}$ and $c_{0}$ on the absolute $\tau$, obtained by the least-squares fitting to Eq. (2). One can see that $\tau$ evidently depends on $a_{0}$ but not on $c_{0}$. (Note that $\tau$ is scaled by neither $a_{0}$ nor $c_{0}$, such as a simple squarepower law). This observation implies that the relaxation along the thickness direction (within the present thickness range) is much faster than that perpendicular to the thickness direction. It has been experimentally shown that the volume change of gels begins at the surfaces for spheres, cylinders, and plates, $, 9,12$ which indicates that the free surface has an important role in determining the kinetics. In the present case, the relaxation in response to a temperature jump is expected to begin at the unclamped side surfaces and can spread to the center portion with time. In a swelling process, the swelling develops from the outer (surface) to the inner (bulk) portions; the preceding (outer) swollen portion is compressed and the delayed (inner) portion is stretched along the one-dimensional thickness direction. Therefore the observed thickness can be determined by the change in the free energy of the system $\Delta G$ resulting from the average contributions of the outer and inner portions. According to the Flory-Huggins theory ${ }^{13} \Delta G$ consists of two contributions of the osmotic term (mixing free energy) $\Delta G_{\text {mix }}$ and the elastic term (elastic energy), $\Delta G_{\mathrm{el}}$. We can estimate $\Delta G$ in the present system by calculating the loss and the gain of $\Delta G_{\mathrm{el}}$ through the following first approximation. Suppose that there is a discrete boundary between the preceding and the delayed portions. If the sum of the changes in the elastic energies of the outer and of the inner portions is zero, one can assume

$$
S\left(c-c_{F}\right)+\left(a_{0} b_{0}-S\right)\left(c-c_{I}\right)=0,
$$

where $S$ denotes the surface area of the preceded portion. This equation results in the thickness change, $c-c_{I}$ $=\left(\Delta c / a_{0} b_{0}\right) S$, which indicates that the time evolution of the thickness should be proportional to $S$. These considerations suggest that the relaxation (single exponential decay) of the thickness is essentially equivalent to the two-dimensional diffusion phenomenon, therefore the swelling and shrinking
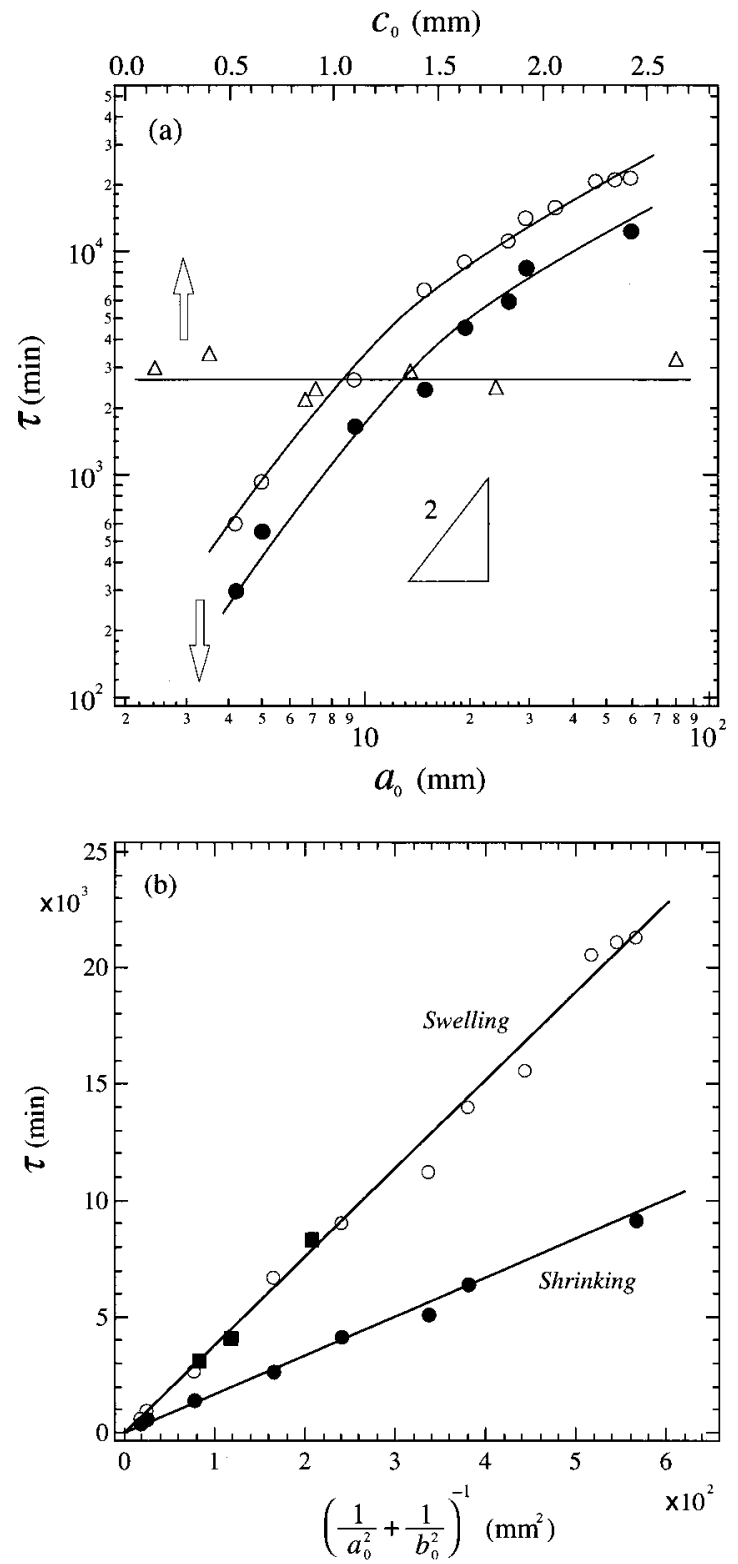

FIG. 3. (a) Characteristic time $\tau$ for the gels with different $a_{0}$ on the swelling (open circle) and the shrinking (closed circle) processes, and for the gels with different $c_{0}$ on the swelling (open triangle). (b) Replot of $\tau$ against $\left(a_{0}^{-2}+b_{0}^{-2}\right)^{-1}$ for the gels with different $a_{0}$. Three data are appended to confirm the model (closed square) by changing $b_{0}$.

kinetics can be determined by the surface lengths $a_{0}$ and $b_{0}$. According to simple two-dimensional diffusion with a square boundary condition, the characteristic time $\tau$ is proportional to a function of the surface lengths $a_{0}$ and $b_{0}$ :

$$
\tau \propto\left(\frac{1}{a_{0}^{2}}+\frac{1}{b_{0}^{2}}\right)^{-1} .
$$

Now we can discuss the effects of the surface lengths on the absolute $\tau$ by using this relation in conjunction with the twodimensional diffusion. In Fig. 3(b), $\tau$ is replotted as a function of the right term of Eq. (4) (open and closed circles). This expression supports the idea that the mechanism of the swelling and shrinking kinetics in the present system obeys the two-dimensional diffusion equation. In order to confirm 
the above consideration $b_{0}$ was also changed to between 12.8 and $20.3 \mathrm{~mm}$. Three examples are appended in Fig. 3(b) (closed square), which successfully show the validity of the model to the present system.

It should be noted that the absolute $\tau$ on shrinking is much smaller than that on swelling. One of the reasons for the smaller $\tau$ in the shrinking process can be attributed to the absolute diffusion constant; $D$ at $30^{\circ} \mathrm{C}$ is larger than $D$ at $11.5^{\circ} \mathrm{C}$. ${ }^{14}$ This is because $\tau$ is expected to be inversely proportional to $D$. However, this evidence might not be good enough to explain the much smaller value on shrinking compared with that on swelling (approximately one second). It is important to take into consideration the possibility of the network orientation that might be introduced at the gelation. During the polymerization the monomers in the pregel solution were first under the influence of thermal fluctuation, and after reaching the gel point the fluctuation became frozen into the gel network. Since the present polymerization is an exothermic reaction, the thermal convection in a thin space between two slides should have an important role in determining the network structure. If the thin-plate gel is oriented vertically, the kinetics should be strongly affected. This is an important subject for future investigations.

It is also worth mentioning that in the kinetics of thinplate gels with the rectangular surfaces, the square-power law between $\tau$ and $a_{0}$ can be realized under two specific conditions. First, the shorter surface length must be negligibly smaller than the longer one $\left(a_{0} \ll b_{0}\right)$. Second, the surface must be a perfect square $\left(a_{0}=b_{0}\right)$. Estimated from Fig. 3 (b), the characteristic time $\tau$ for the gels with a $0.1 \times 0.1$ $\mathrm{mm}^{2}$ surface is roughly $1 \mathrm{~min}$, which is on the same order as the absolute $\tau$ for the cylindrical gels with $0.1 \mathrm{~mm}$ diameter.

\section{CONCLUSIONS}

The swelling and shrinking kinetic of thin-plate NIPA gels under mechanical constraint (the top and bottom sur- faces chemically clamped on the glass plates) was investigated by measuring the macroscopic thickness changes between two swollen states by the temperature jumps. The thickness exponentially approaches the equilibrium value except immediately after the temperature change. We found that the characteristic time is uniquely determined by the lengths of the constrained surfaces, and not by the thickness at gelation. The relaxation is essentially the same as the twodimensional diffusion phenomenon. The present fundamental findings are of crucial importance not only for understanding the nature of the volume change of gels (the development of new science) but also in establishing the foundation for future practical applications of gels.

\section{ACKNOWLEDGMENTS}

This paper is dedicated to the memory of Professor Toyoichi Tanaka of MIT. This work was supported in part by a Grant-in-Aid for Scientific Research from the Ministry of Education, Science, Sports and Culture in Japan, and by a Grant from Nippon Steel Corp.

${ }^{1}$ T. Tanaka and D. J. Fillmore, J. Chem. Phys. 70, 1214 (1979).

${ }^{2}$ T. Tanaka, E. Sato, Y. Hirokawa, S. Hirotsu, and J. Peetermans, Phys. Rev. Lett. 55, 2455 (1985).

${ }^{3}$ T. Tanaka, in Dynamic Light Scattering, edited by R. Pecora (Plenum, New York, 1986), p. 347.

${ }^{4}$ T. Tanaka, Physica A 140, 261 (1986).

${ }^{5}$ A. Peters and S. J. Candau, Macromolecules 19, 1952 (1986).

${ }^{6}$ E. S. Matsuo and T. Tanaka, J. Chem. Phys. 89, 1695 (1988).

${ }^{7}$ Y. Li and T. Tanaka, J. Chem. Phys. 92, 1365 (1990).

${ }^{8}$ A. Suzuki, M. Yamazaki, Y. Kobiki, and H. Suzuki, Macromolecules 30, 2350 (1997)

${ }^{9}$ A. Suzuki, S. Yoshikawa, and G. Bai, J. Chem. Phys. 111, 360 (1999).

${ }^{10}$ Y. Li and T. Tanaka, Annu. Rev. Mater. Sci. 22, 243 (1992).

${ }^{11}$ Y. Hirokawa, H. Jinnai, Y. Nishikawa, T. Okamoto, and T. Hashimoto, Macromolecules 32, 7093 (1999)

${ }^{12}$ S. Hirotsu, J. Chem. Phys. 88, 427 (1988).

${ }^{13}$ P. J. Flory, Principle of Polymer Chemistry (Cornell University Press, Ithaca, 1953).

${ }^{14}$ E. S. Matsuo and T. Tanaka, Phase Transit. 46, 217 (1994). 